\title{
ON EXPECTED DURATIONS OF BIRTH-DEATH PROCESSES, WITH APPLICATIONS TO BRANCHING PROCESSES AND SIS EPIDEMICS
}

FRANK BALL, ${ }^{*}$ University of Nottingham

TOM BRITTON, ${ }^{* *}$ Stockholm University

PETER NEAL,$^{* * *}$ Lancaster University

\footnotetext{
* Postal address: The Mathematical Sciences Building, University Park, Nottingham, NG7 2RD, UK ** Postal address: Department of Mathematics, Stockholm University, SE-106 91 Stockholm, Sweden. *** Postal address: Department of Mathematics and Statistics, Fylde College, Lancaster University,
} LA1 4YF, United Kingdom 


\begin{abstract}
We study continuous-time birth-death type processes, where individuals have independent and identically distributed lifetimes, according to a random variable $Q$, with $\mathrm{E}[Q]=1$, and where the birth rate if the population is currently in state (has size) $n$ is $\alpha(n)$. We focus on two important examples, namely $\alpha(n)=\lambda n$ being a branching process, and $\alpha(n)=\lambda n(N-n) / N$ which corresponds to an SIS (susceptible $\rightarrow$ infective $\rightarrow$ susceptible) epidemic model in a homogeneously mixing community of fixed size $N$. The processes are assumed to start with a single individual, i.e. in state 1 . Let $T, A_{n}, C$ and $S$ denote the (random) time to extinction, the total time spent in state $n$, the total number of individuals ever alive and the sum of the lifetimes of all individuals in the birth-death process, respectively. We give expressions for the expectation of all these quantities and show that these expectations are insensitive to the distribution of $Q$. We also derive an asymptotic expression for the expected time to extinction of the SIS epidemic, but now starting at the endemic state, which is not independent of the distribution of $Q$. The results are also applied to the household SIS epidemic, showing that, in contrast to the household SIR (susceptible $\rightarrow$ infective $\rightarrow$ recovered) epidemic, its threshold parameter $R_{*}$ is insensitive to the distribution of $Q$.

Keywords: Birth-death process; branching processes; SIS epidemics; insensitivity results.

2010 Mathematics Subject Classification: Primary 60J80
\end{abstract}

Secondary 60G10;92D30

\title{
1. Introduction
}

A key question for population processes of a birth-death type, for example, branching processes and epidemic processes (with infection and recovery corresponding to birth and death, respectively), is what effect does the lifetime distribution have on key quantities of scientific interest? For example, consider a single-type branching process, where individuals have independent and identically distributed (iid) lifetimes according to a random variable $Q$ having an arbitrary, but specified, distribution and, whilst alive, give birth at the points of a homogeneous Poisson point process with rate $\lambda$. The basic reproduction number, $R_{0}=\lambda \mathrm{E}[Q]$, the mean number of offspring produced by an individual during its lifetime, depends upon $Q$ only through its mean $\mathrm{E}[Q]$. The mean 
total size of a subcritical branching process $\left(R_{0}<1\right)$ with one ancestor is $1 /\left(1-R_{0}\right)$, which is again independent of the distribution of $Q$. However, other quantities of interest, such as the probability of extinction and the Malthusian parameter of the branching process, depend upon the distributional form of $Q$. Thus, in the language of stochastic networks, $R_{0}$ can be viewed as an insensitivity result in that it depends on $Q$ only through its mean, see, for example, Zachary (2007).

Insensitivity results for stochastic networks are well known, see for example, Sevast'yanov (1957), Whittle (1985) and Zachary (2007). In particular, in Zachary (2007), Theorem 1, it is shown that for a wide class of queueing networks, where arrivals (births) into the system are Poissonian with rate depending upon the total number of individuals in the system and each arrival has an iid workload, the stationary distribution of the total number of individuals in the system is insensitive to the distribution of $Q$. It then follows automatically that, for example, the mean duration of a busy period of the network (at least one individual in the system) is insensitive to the distribution of $Q$.

Given the similarities between queueing networks and birth-death type models, arrivals equating to births and workload equating to lifetime, we seek in this paper to explore insensitivity results for birth-death type processes with particular emphasis upon branching processes and SIS (susceptible $\rightarrow$ infective $\rightarrow$ susceptible) epidemic models. In many cases, Zachary (2007), Theorem 1, cannot be applied directly to birthdeath processes, as many birth-death processes do not exhibit stationary behaviour. For example, a branching process will either go extinct or grow exponentially. However, we can exploit Zachary (2007), Theorem 1, for birth-death type processes whose mean time to extinction is finite by introducing a regeneration step (cf. Hernández-Suárez and Castillo-Chavez (1999)) whenever the population goes extinct. That is, whenever the population goes extinct, it spends an exponential length of time in state 0 (no individuals) before a new individual is introduced into the population (regeneration). The birth-death type process with regenerations then fits into the framework of Zachary (2007), provided that the birth rate is Poissonian and depends upon the population only through its size. Insensitivity results are then easy to obtain for the regenerative process, and also for the original birth-death type process.

The paper is structured as follows. In Section 2, we formally introduce the generic 
birth-death type process with arbitrary birth rate $\alpha(n)$, where $n$ denotes population size, and introduce regeneration. We identify key insensitivity results for birth-death type processes, including, that the mean duration, the mean time with $n$ individuals alive $(n=1,2, \ldots)$ and the mean total number of individuals ever alive in the process are insensitive to the distribution of $Q$. In Section 3, we focus on three special cases of the birth-death type process, namely, branching processes with constant birth rate, and homogeneously mixing and household SIS epidemic models. In Section 3.1, we prove a conjecture of Neal (2014), that for a subcritical branching process, the mean time with $n(n=1,2, \ldots)$ individuals alive is insensitive to $Q$ and, using Lambert (2011), Lemma 3.1, give a corresponding insensitivity result for critical and supercritical branching processes. In Section 3.2, we apply the insensitivity results to homogeneously mixing SIS epidemics and obtain a simple approximation for the mean duration of the epidemic starting from a single infective. Moreover, we show that for a supercritical epidemic $\left(R_{0}>1\right)$, the mean duration of the epidemic starting from the quasi-endemic equilibrium does depend upon the distribution of $Q$ and we give a simple asymptotic expression for this quantity. Finally, in Section 3.3 we exploit the results obtained for the homogeneously mixing SIS epidemic to show that both the threshold parameter $R_{*}$ and the quasi-endemic equilibrium of the household SIS epidemic are insensitive to the distribution of $Q$. These are interesting findings, as in the household SIR (susceptible $\rightarrow$ infective $\rightarrow$ recovered) epidemic both $R_{*}$ and the fraction of the population ultimately recovered if the epidemic takes off do depend upon the distribution of $Q$.

\section{Generic model}

The generic birth-death type process is defined as follows. The process is initiated at time $t=0$ with one individual. All individuals, including the initial individual, have iid lifetimes according to an arbitrary, but specified, positive random variable $Q$ with finite mean. At the end of its lifetime an individual dies and is removed from the population. New individuals are born and enter the population at the points of an independent inhomogeneous Poisson point process with rate $\alpha(n) \geq 0$, where $n$ denotes the total number of individuals in the population. Without loss of generality, 
we assume that $\mathrm{E}[Q]=1$, since otherwise we can simply rescale time by dividing $Q$ and multiplying $\alpha(n)$ by $\mathrm{E}[Q]$. The special cases of a branching process with individuals giving birth at the points of independent homogeneous Poisson point processes with rate $\lambda$ and the homogeneously mixing SIS epidemic (see, for example, Kryscio and Lefèvre (1989)) in a population of size $N$ with infection rate $\lambda$, correspond to $\alpha(n)=n \lambda$ and $\alpha(n)=n \lambda(N-n) / N$, respectively.

The birth-death type process is similar to the single-class networks studied in Zachary (2007), Section 2. In Zachary (2007), it is assumed that new individuals enter the system (births) at the points of a Poisson process with state-dependent rate $\alpha(n)$, where $n$ is the total number of individuals currently in the system. Individuals have iid workloads, according to a random variable $Q$ with $\mathrm{E}[Q]=1$. While there are $n$ individuals in the system, the total workload is reduced at rate $\beta(n) \geq 0$, with $\beta(n)>0$ if and only if $n>0$. In a biological setting, where the workload $Q$ associated with an individual is its lifetime, it only makes sense to take $\beta(n)=n$, so each individual's remaining lifetime decreases at constant rate 1 .

In Zachary (2007), Theorem 1, it is shown that if the proper distribution $\boldsymbol{\pi}=$ $(\pi(0), \pi(1), \ldots)$ satisfies the detailed balance equations

$$
\pi(n+1) \beta(n+1)=\pi(n) \alpha(n), \quad n=0,1, \ldots,
$$

and

$$
\sum_{n=0}^{\infty} \pi(n) \alpha(n)<\infty
$$

then $\pi$ is the stationary distribution of the size of the system, irrespective of the distribution of $Q$.

For many biological systems, Zachary (2007) does not apply since a stationary distribution for the total number of individuals alive does not exist. The solution to make Zachary (2007), Theorem 1, relevant to birth-death type processes is to follow Hernández-Suárez and Castillo-Chavez (1999) and introduce regeneration by setting $\alpha(0)=1$, leaving all other transition rates unchanged. Thus, if the process goes extinct, it spends an exponentially distributed time, having mean 1 , with no individual before a new individual enters the population leading to the process restarting (regeneration). 
Recall that in the processes we study $\beta(n)=n$. Then (2.1) implies that

$$
\pi(n)=\pi(0) \prod_{i=1}^{n-1} \frac{\alpha(i)}{i+1}, \quad n=1,2, \ldots,
$$

where the product is 1 when $n=1$, whence

$$
\pi(0)=\left\{1+\sum_{n=1}^{\infty} \prod_{i=0}^{n-1} \frac{\alpha(i)}{i+1}\right\}^{-1} .
$$

Note that $\boldsymbol{\pi}$ being a proper distribution implicitly implies that $\pi(0)>0$ or, equivalently, that the sum in (2.4) is finite, and hence that the process is positive recurrent. Thus the regenerative process is not suitable for critical or supercritical branching processes. We discuss this in more detail in Section 3.1 below.

We complete this section by identifying a number of key quantities whose means are insensitive to the distribution of $Q$ and are summarised in Theorem 2.1.

Theorem 2.1. Let $Y_{t}$ denote the total number of individuals in the birth-death process at time t. Let $T=\int_{0}^{\infty} 1_{\left\{Y_{t}>0\right\}} d t$ denote the duration of the birth-death process and, for $n=1,2, \ldots$, let $A_{n}=\int_{0}^{\infty} 1_{\left\{Y_{t}=n\right\}} d t$ denote the total time the birth-death process spends with $n$ individuals alive. Then

$$
\mathrm{E}[T]=\sum_{n=1}^{\infty} \prod_{i=0}^{n-1} \frac{\alpha(i)}{i+1}
$$

and for $n=1,2, \ldots$,

$$
\mathrm{E}\left[A_{n}\right]=\prod_{i=0}^{n-1} \frac{\alpha(i)}{i+1} .
$$

Finally, let $C$ denote the total number of individuals ever alive in the birth-death process and let $S$ be the sum of the lifetimes of those $C$ individuals. Then

$$
\mathrm{E}[C]=\mathrm{E}[S]=\sum_{k=1}^{\infty} k \prod_{i=1}^{k-1} \frac{\alpha(i)}{i+1} .
$$

Proof. An immediate consequence of the above construction is that the mean time between regenerations is $1 / \pi(0)$, irrespective of the distribution of $Q$. On average one unit of time is spent with no individual in the population, so (see Ball and Milne (2004) for a formal justification)

$$
\mathrm{E}[T]=\frac{1}{\pi(0)}-1=\left\{\sum_{n=1}^{\infty} \prod_{i=0}^{n-1} \frac{\alpha(i)}{i+1}\right\},
$$


as required. Moreover, using (2.3), for $n=1,2, \ldots$,

$$
\mathrm{E}\left[A_{n}\right]=\pi(n) \frac{1}{\pi(0)}=\prod_{i=0}^{n-1} \frac{\alpha(i)}{i+1} .
$$

Using Fubini's theorem,

$$
\begin{aligned}
\mathrm{E}[S] & =\mathrm{E}\left[\int_{0}^{\infty} Y_{t} d t\right]=\mathrm{E}\left[\int_{0}^{\infty} \sum_{k=1}^{\infty} k 1_{\left\{Y_{t}=k\right\}} d t\right]=\sum_{k=1}^{\infty} k \mathrm{E}\left[\int_{0}^{\infty} 1_{\left\{Y_{t}=k\right\}} d t\right] \\
& =\sum_{k=1}^{\infty} k \mathrm{E}\left[A_{k}\right]=\sum_{k=1}^{\infty} k \prod_{i=1}^{k-1} \frac{\alpha(i)}{i+1} .
\end{aligned}
$$

Note that, after using (2.1) with $\beta(n)=n,(2.2)$ ensures that $\mathrm{E}[S]$ is finite.

Given $A_{k}$, the mean number of births whilst the process is in state $k$ is $\alpha(k) A_{k}$, so, including the initial ancestor and noting from (2.9) that $\mathrm{E}\left[A_{1}\right]=1$, we have that

$$
\begin{aligned}
\mathrm{E}[C] & =\mathrm{E}\left[1+\sum_{k=1}^{\infty} \alpha(k) A_{k}\right]=1+\sum_{k=1}^{\infty} \alpha(k) \frac{\pi(k)}{\pi(0)}=\mathrm{E}\left[A_{1}\right]+\sum_{k=1}^{\infty}(k+1) \frac{\pi(k+1)}{\pi(0)} \\
& =\mathrm{E}\left[A_{1}\right]+\sum_{k=2}^{\infty} k \mathrm{E}\left[A_{k}\right] \\
& =\mathrm{E}[S] .
\end{aligned}
$$

\section{Special cases}

\subsection{Branching process}

As mentioned above, we consider branching processes where individuals have iid lifetimes according to $Q$ (with $E[Q]=1$ ) and whilst alive give birth at the points of independent homogeneous Poisson point processes with rate $\lambda$. Therefore we have that $\alpha(n)=n \lambda$. The key result is Lemma 3.1, which is a generalization of Neal (2014), Conjecture 2.1.

Lemma 3.1. For $n=1,2, \ldots$,

$$
\mathrm{E}\left[A_{n}\right]=\frac{\lambda^{n-1}}{n(\max \{1, \lambda\})^{n}} .
$$

The Lemma is proved in (3.5) and (3.8) below.

For $\lambda<1$, the branching process is subcritical and the results of Section 2 hold. It follows from (2.3) that

$$
\pi(n)=\pi(0) \prod_{i=1}^{n-1} \frac{i \lambda}{i+1}=\pi(0) \frac{\lambda^{n-1}}{n}, \quad n=1,2, \ldots,
$$


whence

$$
\pi(0)=\left\{1+\sum_{n=1}^{\infty} \frac{\lambda^{n-1}}{n}\right\}^{-1}=\{1-\log (1-\lambda) / \lambda\}^{-1} .
$$

Therefore, it follows from (3.3) and (2.5) that the mean duration of the branching process is

$$
\mathrm{E}[T]=-\log (1-\lambda) / \lambda
$$

Also, Neal (2014), Conjecture 2.1, is proved in that, for $n=1,2, \ldots$,

$$
\mathrm{E}\left[A_{n}\right]=\frac{\lambda^{n-1}}{n} .
$$

Finally, we obtain the classical result that the mean total number of individuals ever alive in the branching process is

$$
\mathrm{E}[C]=\sum_{k=1}^{\infty} k \mathrm{E}\left[A_{k}\right]=\sum_{k=1}^{\infty} \lambda^{k-1}=\frac{1}{1-\lambda} .
$$

The above arguments break down when $\lambda \geq 1$, since then the sum in (3.3) diverges. However, progress can be made in extending Neal (2014), Conjecture 2.1, and (3.5) to this case by using Lambert (2011), Lemma 3.1. (Note that the mean duration and mean total number of individuals ever alive in the branching process are now both infinite.) Specifically, Lambert (2011), Lemma 3.1, shows that, for $n=1,2, \ldots$,

$$
\mathrm{P}\left(Y_{t}=n\right)=\left(1-\frac{1}{W(t)}\right)^{n-1} \frac{W^{\prime}(t)}{\lambda W(t)^{2}}, \quad t \geq 0,
$$

where $W(t)$ solves Lambert (2011), equation (6). For most choices of $Q$, it is not possible to get an explicit expression for $W(t)$ for all $t \geq 0$. However, for any $Q$ $(\mathrm{E}[Q]=1) W(0)=1$ and for $\lambda \geq 1, W(\infty)=\infty$. Therefore, for $n=1,2, \ldots$,

$$
\begin{aligned}
\mathrm{E}\left[A_{n}\right]=\int_{0}^{\infty} \mathrm{P}\left(Y_{t}=n\right) d t & =\frac{1}{\lambda} \int_{0}^{\infty}\left(1-\frac{1}{W(t)}\right)^{n-1} \frac{W^{\prime}(t)}{W(t)^{2}} d t \\
& =\frac{1}{\lambda}\left[\frac{1}{n}\left(1-\frac{1}{W(t)}\right)^{n}\right]_{0}^{\infty}=\frac{1}{\lambda n}
\end{aligned}
$$

Note that if $\lambda<1, W(\infty)=1 /(1-\lambda)$ and (3.8) can be used to obtain (3.5) directly.

\subsection{SIS epidemic}

3.2.1. Mean duration with one initial infective As noted in Section 2, setting $\alpha(n)=$ $\lambda n(N-n) / N(n=1,2, \ldots, N)$ yields a homogeneously mixing SIS epidemic in a 
population of size $N$. Using (2.3), it is straightforward to show that, for $n=1,2, \ldots, N$,

$$
\pi^{(N)}(n)=\frac{\pi^{(N)}(0)}{n} \frac{(N-1) !}{(N-n) !}\left(\frac{\lambda}{N}\right)^{n-1}
$$

which has previously been obtained for the Markov case $(Q \sim \operatorname{Exp}(1))$ by HernándezSuárez and Castillo-Chavez (1999). Consequently, the mean duration of the epidemic starting from a single infective is

$$
\mathrm{E}\left[T^{(N)}\right]=\sum_{n=1}^{N} \frac{(N-1) !}{n(N-n) !}\left(\frac{\lambda}{N}\right)^{n-1} .
$$

Note that this follows directly from (2.5), on recalling that there $\alpha(0)=1$. We index quantities of interest by the total population size $N$ to highlight the role played by $N$ in the analysis below, where we investiage their behaviour for large $N$.

Lemma 3.2. For the subcritical case, $\lambda<1$,

$$
\mathrm{E}\left[T^{(N)}\right] \rightarrow-\frac{\log (1-\lambda)}{\lambda} \quad \text { as } N \rightarrow \infty .
$$

For the critical case, $\lambda=1$,

$$
\mathrm{E}\left[T^{(N)}\right] \sim \frac{1}{2} \log N
$$

where $a^{(N)} \sim b^{(N)}$ denotes that $\lim _{N \rightarrow \infty} a^{(N)} / b^{(N)}=1$.

For the supercritical case, $\lambda>1$,

$$
\mathrm{E}\left[T^{(N)}\right] \sim \frac{\sqrt{2 \pi}}{\lambda-1} \frac{\exp (\{\log \lambda-1+1 / \lambda\} N)}{\sqrt{N}} .
$$

Proof. First note from (3.10) that

$$
\mathrm{E}\left[T^{(N)}\right]=\sum_{n=1}^{N} \prod_{i=1}^{n-1}\left(1-\frac{i}{N}\right) \frac{\lambda^{n-1}}{n} .
$$

Suppose that $\lambda<1$. Then, for any $k \in \mathbb{N}$,

$$
\liminf _{N \rightarrow \infty} \mathrm{E}\left[T^{(N)}\right] \geq \liminf _{N \rightarrow \infty} \sum_{n=1}^{k} \prod_{i=1}^{n-1}\left(1-\frac{i}{N}\right) \frac{\lambda^{n-1}}{n}=\sum_{n=1}^{k} \frac{\lambda^{n-1}}{n},
$$

and letting $k \rightarrow \infty$ yields

$$
\liminf _{N \rightarrow \infty} \mathrm{E}\left[T^{(N)}\right] \geq-\frac{\log (1-\lambda)}{\lambda} .
$$


Also, for any $k \in \mathbb{N}$,

$\limsup _{N \rightarrow \infty} \mathrm{E}\left[T^{(N)}\right] \leq \limsup _{N \rightarrow \infty} \sum_{n=1}^{k} \prod_{i=1}^{n-1}\left(1-\frac{i}{N}\right) \frac{\lambda^{n-1}}{n}+\sum_{n=k+1}^{\infty} \lambda^{n-1}=\sum_{n=1}^{k} \frac{\lambda^{n-1}}{n}+\frac{\lambda^{k}}{1-\lambda}$,

and letting $k \rightarrow \infty$ yields

$$
\limsup _{N \rightarrow \infty} \mathrm{E}\left[T^{(N)}\right] \leq-\frac{\log (1-\lambda)}{\lambda} .
$$

Combining (3.15) and (3.16) yields (3.11).

Suppose that $\lambda=1$. Then, setting $\lambda=1$ in (3.14) and noting that

$$
\prod_{i=1}^{n-1}\left(1-\frac{i}{N}\right) \leq \prod_{i=1}^{n-1} \exp \left(-\frac{i}{N}\right)=\exp \left(-\frac{n(n-1)}{2 N}\right)
$$

yields that, for any $L>0$ and all sufficiently large $N$,

$\mathrm{E}\left[T^{(N)}\right] \leq \sum_{n=1}^{\lceil L \sqrt{N}\rceil} \frac{1}{n}+\sum_{\lceil L \sqrt{N}\rceil+1}^{N} \frac{1}{n} \exp \left(-\frac{n(n-1)}{2 N}\right) \leq \sum_{n=1}^{\lceil L \sqrt{N}\rceil} \frac{1}{n}+\exp \left(-L^{2} / 2\right) \sum_{\lceil L \sqrt{N}\rceil+1}^{N} \frac{1}{n}$,

where, for $x \in(R),\lceil x\rceil$ denotes the smallest integer $\geq x$. Hence,

$$
\limsup _{N \rightarrow \infty} \frac{\mathrm{E}\left[T^{(N)}\right]}{\frac{1}{2} \log N} \leq 1+\exp \left(-L^{2} / 2\right) .
$$

Setting $\lambda=1$ in (3.14) yields that, for any $K>0$ and all sufficiently large $N$,

$$
\mathrm{E}\left[T^{(N)}\right] \geq \sum_{n=1}^{\lceil K \sqrt{N}\rceil} \frac{1}{n} \prod_{i=1}^{n-1}\left(1-\frac{i}{N}\right) \geq\left(\sum_{n=1}^{\lceil K \sqrt{N}\rceil} \frac{1}{n}\right)_{i=1}^{\lceil K \sqrt{N}\rceil}\left(1-\frac{i}{N}\right) .
$$

Straightforward analysis (note the connection with the birthday problem and Aldous (1985), page 96) yields

$$
\lim _{N \rightarrow \infty} \prod_{i=1}^{\lceil K \sqrt{N}\rceil}\left(1-\frac{i}{N}\right)=\exp \left(-K^{2} / 2\right),
$$

whence

$$
\liminf _{N \rightarrow \infty} \frac{\mathrm{E}\left[T^{(N)}\right]}{\frac{1}{2} \log N} \geq \exp \left(-K^{2} / 2\right) .
$$

Letting $L \rightarrow \infty$ in (3.17) and $K \downarrow 0$ in (3.18) yields (3.12).

For $\lambda>1$, rearranging (3.10) yields $\mathrm{E}\left[T^{(N)}\right]=a^{(N)} b^{(N)}$, where $a^{(N)}=\frac{N !}{N^{2}}(\lambda / N)^{N-1} \mathrm{e}^{N / \lambda}$ and $b^{(N)}=N \mathrm{e}^{-N / \lambda} \sum_{j=0}^{N-1} \frac{(N / \lambda)^{j} \mathrm{e}^{-N / \lambda}}{(N-j) j !}$. A simple calculation using Stirling's approximation, i.e. $N ! \sim \sqrt{2 \pi N}(N / \mathrm{e})^{N}$, yields $a^{(N)} \sim \frac{\sqrt{2 \pi}}{\lambda \sqrt{N}} \exp (\{\log \lambda-1+1 / \lambda\} N)$, so to complete the proof of (3.13) we show that $b^{(N)} \sim \lambda /(\lambda-1)$. 
Fix $\epsilon \in\left(0, \lambda^{-1}\right)$ and let $A_{1}^{\epsilon}=\left\{j \in \mathbb{Z}: 0 \leq j<N\left(\lambda^{-1}-\epsilon\right)\right\}, A_{2}^{\epsilon}=\{j \in \mathbb{Z}$ : $\left.N\left(\lambda^{-1}-\epsilon\right) \leq j \leq N\left(\lambda^{-1}+\epsilon\right)\right\}$ and $A_{3}^{\epsilon}=\left\{j \in \mathbb{Z}: N\left(\lambda^{-1}+\epsilon\right)<j \leq N-1\right\}$. Further, let $X^{(N)}$ denote a Poisson random variable with mean $N / \lambda$. Then, using Chebyshev's inequality, $\mathrm{P}\left(X^{(N)} \in A_{1}^{\epsilon}\right) \rightarrow 0$ and $\mathrm{P}\left(X^{(N)} \in A_{2}^{\epsilon}\right) \rightarrow 1$ as $N \rightarrow \infty$. Also, by large deviation theory, there exists $a>0$, independent of $N$, such that $\mathrm{P}\left(X^{(N)}>N\left(\lambda^{-1}+\epsilon\right)\right) \leq \mathrm{e}^{-a N}$. Now

$$
N \sum_{j \in A_{1}^{\epsilon}} \frac{(N / \lambda)^{j} \mathrm{e}^{-N / \lambda}}{(N-j) j !} \leq \frac{1}{\lambda^{-1}-\epsilon} \mathrm{P}\left(X^{(N)} \in A_{1}^{\epsilon}\right) \rightarrow 0 \quad \text { as } \quad N \rightarrow \infty
$$

and

$$
N \sum_{j \in A_{3}^{\epsilon}} \frac{(N / \lambda)^{j} \mathrm{e}^{-N / \lambda}}{(N-j) j !} \leq N \mathrm{P}\left(X^{(N)}>N\left(\lambda^{-1}+\epsilon\right)\right) \rightarrow 0 \quad \text { as } \quad N \rightarrow \infty .
$$

Also,

$$
\frac{1}{1-\lambda^{-1}+\epsilon} \mathrm{P}\left(X^{(N)} \in A_{2}^{\epsilon}\right) \leq N \sum_{j \in A_{2}^{\epsilon}} \frac{(N / \lambda)^{j} \mathrm{e}^{-N / \lambda}}{(N-j) j !} \leq \frac{1}{1-\lambda^{-1}-\epsilon} \mathrm{P}\left(X^{(N)} \in A_{2}^{\epsilon}\right),
$$

whence, using (3.19), (3.20) and $\lim _{N \rightarrow \infty} \mathrm{P}\left(X^{(N)} \in A_{2}^{\epsilon}\right)=1$,

$$
\liminf _{N \rightarrow \infty} b^{(N)} \geq \frac{1}{1-\lambda^{-1}+\epsilon} \quad \text { and } \quad \limsup _{N \rightarrow \infty} b^{(N)} \leq \frac{1}{1-\lambda^{-1}-\epsilon} .
$$

Letting $\epsilon \downarrow 0$ in the two inequalities in (3.21) yields $b^{(N)} \sim \lambda /(\lambda-1)$, as required.

Let $A_{n}^{(N)}$ denote the total amount of time that the SIS epidemic, initiated with a single infective, spends with $n$ infectious individuals. Then, from (2.9) and (2.8),

$$
\mathrm{E}\left[A_{n}^{(N)}\right]=\frac{\pi^{(N)}(n)}{\pi^{(N)}(0)}=\frac{\pi^{(N)}(n)}{1-\pi^{(N)}(0)} \mathrm{E}\left[T^{(N)}\right] .
$$

Using (2.11), the first equation in (3.22) and (3.9), the mean total number of infectives during the course of a supercritical epidemic is

$$
\begin{aligned}
\mathrm{E}\left[C^{(N)}\right]=\sum_{n=1}^{N} n \mathrm{E}\left[A_{n}^{(N)}\right] & =\sum_{n=1}^{N} n \frac{\pi^{(N)}(n)}{\pi^{(N)}(0)} \\
& =\sum_{n=1}^{N} \frac{(N-1) !}{(N-n) !}\left(\frac{\lambda}{N}\right)^{n-1} \\
& \sim \frac{\sqrt{2 \pi}}{\lambda} \sqrt{N} \exp (\{\log \lambda-1+1 / \lambda\} N) .
\end{aligned}
$$

The derivation of (3.24) is similar to but simpler than that of $\mathrm{E}\left[T^{(N)}\right]$. 
Note that the second equation in (3.22) gives

$$
\mathrm{E}\left[C^{(N)}\right]=\left(\sum_{n=1}^{N} \frac{n \pi^{(N)}(n)}{1-\pi^{(N)}(0)}\right) \mathrm{E}\left[T^{(N)}\right] .
$$

The distribution $\tilde{\boldsymbol{\pi}}^{(N)}=\left(\tilde{\pi}_{1}^{(N)}, \tilde{\pi}_{2}^{(N)}, \ldots, \tilde{\pi}_{N}^{(N)}\right)$, where $\tilde{\pi}_{n}^{(N)}=\pi^{(N)}(n) /\left(1-\pi^{(N)}(0)\right)$, gives a "quasi-equilibrium" distribution for the SIS epidemic. Thus, the mean total number of infectives in the epidemic is given by the mean number of infectives in quasiequilibrium multiplied by the mean duration of the epidemic. When the epidemic is supercritical $(\lambda>1)$, the distribution of $\tilde{\boldsymbol{\pi}}^{(N)}$ is concentrated on values close to $N\left(1-\lambda^{-1}\right)$, which explains the simple multiplicative relationship between the approximations (3.13) and (3.24).

3.2.2. Mean extinction time from quasi-endemic equilibrium The above calculations of $\mathrm{E}\left[T^{(N)}\right]$ are insensitive to the distribution of $Q$. However, for supercritical SIS epidemics there is interest in the time to extinction of the epidemic starting from the quasi-endemic equilibrium of around $N(1-1 / \lambda)$ infectives. We outline how the mean time to extinction from the quasi-endemic equilibrium, $\mathrm{E}\left[T_{Q}^{(N)}\right]$, does depend upon the distribution of $Q$. The epidemic initiated from a single infective either goes extinct very quickly or takes off and reaches an endemic equilibrium of a proportion $(\lambda-1) / \lambda$ of the population infected, cf. Kryscio and Lefèvre (1989). The epidemic then spends a long time fluctuating about the endemic equilibrium before eventually going extinct. This can be seen from $\boldsymbol{\pi}^{(N)}$, with most of the probability mass centred about $(\lambda-1) N / \lambda$ infectives. There has been considerable interest in investigating the distribution of the time to extinction from the endemic equilibrium, see for example Kryscio and Lefèvre (1989), Andersson and Djehiche (1998), Nåsell (1999) and Britton and Neal (2010). This is a difficult problem on which to make analytical progress. In Andersson and Djehiche (1998), it was shown that, when $Q \sim \operatorname{Exp}(1)$, the time to extinction divided by $\mathrm{E}\left[T_{Q}^{(N)}\right]$ converges in distribution to $\operatorname{Exp}(1)$ as $N \rightarrow \infty$. Moreover, $\mathrm{E}\left[T_{Q}^{(N)}\right] \sim \sqrt{2 \pi / N} \lambda \exp (N\{\log \lambda+1 / \lambda-1\}) /(\lambda-1)^{2}=\mu^{(N)} /(1-1 / \lambda)$, where $\mu^{(N)}=\mathrm{E}\left[T^{(N)}\right]$. It is conjectured that an exponential distribution for the time to extinction holds more generally than for $Q \sim \operatorname{Exp}(1)$, but even computing $\mathrm{E}\left[T_{Q}^{(N)}\right]$ up to leading terms in $N$ has proved difficult. By studying Gaussian approximations for the endemic equilibrium, qualitative results on the time to extinction have been 
obtained, see Nåsell (1999) and Britton and Neal (2010). Whilst, such approaches have given a qualitative understanding of extinction of SIS epidemics, the estimates obtained for the mean time to extinction are incorrect by orders of magnitude. Moreover, it is noted in Neal (2014) that simulation results suggest that the distribution of $Q$ does affect the mean time to extinction from the quasi-endemic equilibrium, which is not predicted by using the qualitative Gaussian approximation.

Lemma 3.3. For $\lambda>1$ and $\operatorname{var}(Q)<\infty$,

$$
\mathrm{E}\left[T_{Q}^{(N)}\right] \sim \frac{1}{1-p_{Q}} \mu^{(N)},
$$

where $p_{Q}$ is the extinction probability of the branching process studied in Section 3.1.

The requirement that $\operatorname{var}(Q)<\infty$ is almost certainly not necessary but is assumed in the formal proof of Lemma 3.3, which is very long and will be presented elesewhere. Here we give a heuristic proof. As noted above, the supercritical SIS epidemic will either quickly go extinct or will take-off and reach the endemic equilibrium. Let $1-P_{Q}^{(N)}$ denote the probability that the number of infectives $Y_{t}$ is at least $\log N$ at some point in time. Then it is straightforward to show, using a branching process approximation (see, for example, Whittle (1955) and Ball and Donnelly (1995)), that $P_{Q}^{(N)} \rightarrow p_{Q}$ as $N \rightarrow \infty$. Then the mean duration of an epidemic, initiated from a single infective satisfies

$$
\mu^{(N)}=\frac{1}{\pi^{(N)}(1)}=P_{Q}^{(N)} A_{Q}^{(N)}+\left(1-P_{Q}^{(N)}\right)\left\{B_{Q}^{(N)}+\mathrm{E}\left[T_{Q}^{(N)}\right]\right\},
$$

where $A_{Q}^{(N)}$ is the mean duration of an epidemic which never reaches $\log N$ infected (epidemic dies off quickly) and $B_{Q}^{(N)}$ is the mean time take for the epidemic to reach the endemic equilibrium given it reaches $\log N$ infected. The definition of $B_{Q}^{(N)}$ is imprecise and correspondingly we take $B_{Q}^{(N)}$ to be the mean time to reach at least $(\lambda-1) N / \lambda$ infectives given that the epidemic takes off. For the case $Q \sim \operatorname{Exp}(1)$, it is shown in Andersson and Djehiche (1998) that $A_{Q}^{(N)}=O(1)$ and $B_{Q}^{(N)}=O(\log N)$. Therefore, assuming that $A_{Q}^{(N)}$ and $B_{Q}^{(N)}$ are both $o\left(\mu^{(N)}\right)$ for general $Q$, we have that

$$
\begin{aligned}
\mathrm{E}\left[T_{Q}^{(N)}\right] & =\frac{1}{1-P_{Q}^{(N)}}\left\{\frac{1}{\pi^{(N)}(1)}-P_{Q}^{(N)} A_{Q}^{(N)}-\left(1-P_{Q}^{(N)}\right) B_{Q}^{(N)}\right\} \\
& \sim \frac{1}{1-p_{Q}} \times \frac{1}{\pi^{(N)}(1)}=\frac{1}{1-p_{Q}} \mu^{(N)}
\end{aligned}
$$


which highlights the role of $Q$ in $\mathrm{E}\left[T_{Q}^{(N)}\right]$. Specifically, the greater the extinction probability $p_{Q}$, the longer the epidemic will on average persist, given that it takes off and becomes established. Note that, subject to $\mathrm{E}[Q]=1, p_{Q}$ is least when $Q$ is constant (i.e. $\mathrm{P}(Q=1)=1$ ), so the model with a constant infectious period has the shortest mean time to extinction starting from quasi-endemic equilibrium.

\subsection{Household SIS epidemic}

Consider a fixed community consisting of $m$ households which, for simplicity of exposition, all have the same size $h$, so the population size is $N_{h}=m h$. Our results extend straightforwardly to the case where the household sizes are unequal. We are particularly interested in the case where $m$, and hence $N_{h}$, is large. Infectious individuals have iid infectious periods according to $Q$, after which they become susceptible again. While infectious an individual makes two types of contacts: the individual makes global infectious contacts at rate $\lambda_{G}$, each time the contacted person is selected independently and uniformly at random from the whole community, including individuals in the same household, and the individual makes local infectious contacts at rate $(h-1) \lambda_{L}$, each time the contacted person is selected independently and uniformly at random from the other $h-1$ individuals in the same household. If a contacted person is susceptible then he/she becomes infected, otherwise nothing happens. By examining the within-household dynamics of the SIS epidemic in the initial stages of the epidemic and at the quasi-endemic equilibrium, we obtain interesting, and perhaps unexpected, insensitivity results for the household SIS epidemic model.

For large $m$, the initial stages of the household SIS epidemic can be approximated by a branching process; see Ball (1999), where the approximation is made fully rigorous using a coupling argument. The branching process approximation is similar to that used for the household SIR epidemic, Ball et al. (1997), with individuals in the approximating branching process corresponding to within-household epidemic outbreaks in the epidemic. For large $m$, in the initial stages of the household SIS epidemic the probability that a global infectious contact is with an infectious household (a household containing at least one infective) is very small. Therefore, we assume that all global infectious contacts are with totally susceptible households and we consider the epidemic within a household, ignoring for the moment global infectious contacts, initiated by a 
single infective and without any additional global infections from outside.

Let $S$ denote the severity of such a within-household epidemic, i.e. the sum of the infectious periods of all infectives during the course of the epidemic from the initial infective until the epidemic within the household ceases. Then, conditional upon $S$, the total number of global infectious contacts emanating from the household has a Poisson distribution with mean $\lambda_{G} S$, so the basic reproduction number of the approximating branching process is $R_{*}=\lambda_{G} \mathrm{E}[S]$. The within-household epidemic without additional global infections is simply a homogeneously mixing SIS epidemic with $N=h$ and $\lambda / N=\lambda_{L}$, so $\lambda(n)=\lambda_{L} n(h-n)$, and (2.12) and (3.23) imply that

$$
R_{*}=\lambda_{G} \sum_{n=1}^{h} \frac{(h-1) !}{(h-n) !} \lambda_{L}^{n-1},
$$

irrespective of the distribution of $Q$. Note, however, that the distribution of $S$ does depend upon the distribution of $Q$, and hence so does the probability that the epidemic takes off, corresponding to the approximating branching process not going extinct.

Suppose that $R_{*}>1$ and that the epidemic takes off. Its quasi-endemic equilibrium can be determined as follows; see Ghoshal et al. (2004) and Neal (2006). Suppose that a proportion $s$ of the population is infected and focus on a single household. Then each susceptible individual in that household receives global infectious contacts at the points of a homogeneous Poisson process with rate $\lambda_{G} s$, so the number of infectives in that household follows a birth-death process with birth rate $\alpha(n)=(h-n)\left\{s \lambda_{G}+n \lambda_{L}\right\}$. Note that this birth-death process is stationary and denote its equilibrium distribution by $\phi(s)=\left(\phi_{0}(s), \phi_{1}(s), \ldots, \phi_{h}(s)\right)$. It follows, using (2.1) with $\beta(n)=n$, that

$$
\phi_{k}(s)=\frac{\prod_{i=0}^{k-1}\left(\frac{h-i}{i+1}\right)\left(\lambda_{G} s+i \lambda_{L}\right)}{1+\sum_{j=1}^{h} \prod_{i=0}^{j-1}\left(\frac{h-i}{i+1}\right)\left(\lambda_{G} s+i \lambda_{L}\right)}, \quad k=0,1, \ldots, h .
$$

Let $z$ be the proportion of the population that are infected at the quasi-endemic equilibrium. Then, by a self-consistency argument, the mean of the distribution $\phi(z)$ must be $h z$, so $z$ satisfies

$$
h z=\sum_{i=0}^{h} i \phi_{i}(z)
$$

which has a unique strictly positive solution if $R_{*}>1$; see Neal (2006), where the argument is made fully rigorous in the limit as $m \rightarrow \infty$. Note that, for $s>0$, the 
distribution $\phi(s)$ is insensitive to the distribution of $Q$. Hence, at the endemic equilibrium of the household SIS epidemic, both the proportion of the population infected and the distribution of the number infected in a typical household are insensitive to the distribution of $Q$.

The above results are in sharp contrast to the household SIR epidemic, in which the threshold parameter $R_{*}$, the fraction of the population infected by a major outbreak and the distribution of the ultimate number of susceptibles in a typical household in the event of a major outbreak are all sensitive to the distribution of $Q$.

\section{Acknowledgements}

We would like to thank Amaury Lambert for bringing Lambert (2011) and the proof of (3.8) to our attention. Tom Britton is grateful to the Swedish Research Council for financial support.

\section{References}

Aldous, D. J. (1985) Exchangeability and related topics. In Ecole d'Été de Probabilités de Saint-Flour XIII, 1993 (Lecture Notes in Mathematics 1117), ed. Hennequin, P. L. Springer, Berlin.

Andersson, H. and Djehiche, B. (1998) A threshold limit theorem for the stochastic logistic epidemic. J. Appl. Prob. 35, 662-670.

Ball, F. (1999). Stochastic and deterministic models for SIS epidemics among a population partitioned into households. Math. Biosci., 156, 41-67.

Ball, F.G. and Donnelly, P. (1995) Strong approximations for epidemic models. Stoch. Proc. Appl., 55, 1-21.

Ball, F.G. and Milne, R.K. (2004) Applications of simple point process methods to superpositions of aggregated stationary processes. Aust. N. Z. J.Stat., 46, 181-196.

Ball, F., Mollison, D. and Scalia-Tomba, G. (1997). Epidemics with two levels of mixing. Ann. Appl Probab., 7, 46-89. 
Britton, T. and Neal, P. (2010) The time to extinction for an SIS-household-epidemic model. J. Math. Biol., 61, 763-779.

Ghoshal, G. Sander, L. and Sokolov, I. (2004). SIS epidemics with household structure: the self-consistent field method. Math. Biosci., 190, 71-85.

Hernández-Suárez, C.M. and Castillo-Chavez, C. (1999) A basic result on the integral for birth-death Markov processes. Math Biosci., 161, 95-104.

Kryscio, R. and Lefèvre, C. (1989). On the extinction of the SIS stochastic logistic epidemic. J. Appl. Prob., 26, 685-694.

Lambert, A. (2011) Species abundance distributions in neutral models with immigration or mutation and general lifetimes. J. Math. Biol., 63, 57-72.

Nåsell, I. (1999) On the time to extinction in recurrent epidemics. J. R. Stat. Soc. Ser. B Stat. Methodol., 61, 309-330.

Neal, P. (2006) Stochastic and deterministic analysis of SIS household epidemics. Adv. Appl. Prob. 38, 943-968. (See also correction in Adv. Appl. Prob. 44, 309310 (2012).)

Neal, P. (2014) Endemic behaviour of SIS epidemics with general infectious period distributions. Adv. Appl. Prob., 46, 241-255.

Sevast'yanov, B.A. (1957) An ergodic theory for Markov processes and its application to a telephone system with refusals. Theory Prob. Appl., 2, 104-112.

Whittle, P. (1955) The outcome of a stochastic epidemic - a note on Bailey's paper. Biometrika, 42, 116-122.

Whittle, P. (1985) Partial balance and insensitivity. J. Appl. Probab., 22, 168-176.

Zachary, S. (2007). A note on insensitivity in stochastic networks. J. Appl. Probab., 44, 238-248. 